\title{
Quantum Computing in Business Applications is coming
}

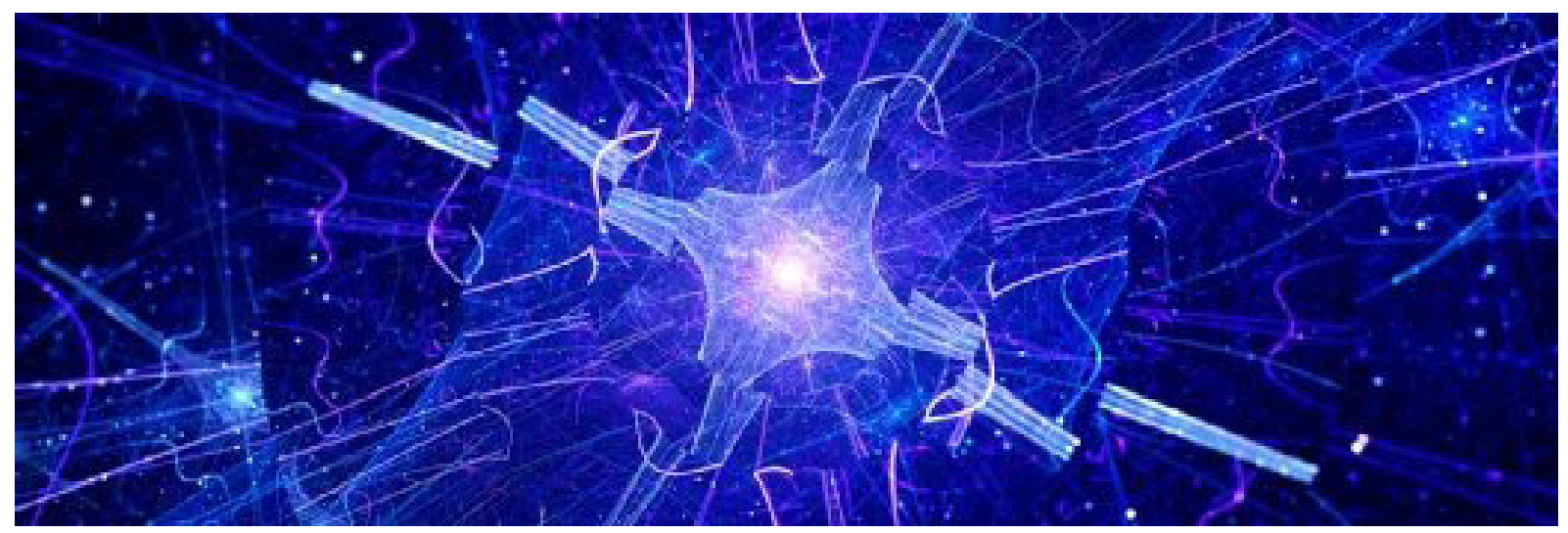

Quantum computers are closer than you think. While tech companies make progress toward building functioning machines, there are quantum applications that organizations can utilize.

Quantum computing may still be in its infancy stages, but it's rapidly approaching and is already showing significant potential for business applications across several industries.

At MIT Technology Review's Future Compute conference in December 2019, Alan Baratz, CEO of D-Wave Systems Inc., a Canadian quantum computing company, discussed the benefits of quantum computing in business applications and the new capabilities it can offer. Editor's note: The following has been edited for clarity and brevity.

Why should $\mathrm{ClOs}$ be thinking about quantum computing in business applications?

Alan Baratz: Quantum computing can accelerate the time to solve the hard problems. If you are in a logistics business and you need to worry about pack and ship or vehicle scheduling; or you're in aerospace and you need to worry about crew or flight scheduling; or a drug company and you need to worry about molecular discovery computational chemistry, the compute time to solve those problems at scale can be very large.
Typically, what companies do is come up with heuristics -- they try to simplify the problem. Well, quantum computing has the potential to allow you to solve the complete problem much faster to get better solutions and optimize your business.

Would you say speed is considered as the most significant factor of quantum computing?

Baratz: Well, sometimes it's speed, sometimes it's quality of the solution [or] better solution within the same amount of time. Sometimes, it's diversity of solution. One of the interesting things about the quantum computer is that maybe you don't necessarily want the optimal solution but [rather] a set of good solutions that you can then use to optimize other things that weren't originally a part of the problem. The quantum computer is good at giving you a set of solutions that are close to optimal in addition to the optimal solution.

What's limiting quantum computing in terms of hardware?

Baratz: Well, up until now, in [D-Wave's] case, it's been the size and topology of the system 
because in order to solve your problem, you have to be able to map it onto the quantum processor. Remember, we aren't gate-based, so it's not like you specify the sequence of instructions or the gates. In order to solve your problem in our system, you have to take the whole problem and be able to map it into our hardware. That means with 2,000 qubits and each qubit connected to six others, there's only certain size problems that you can actually map into our system. When we deliver the Advantage system next year, we double that -- over 5,000 qubits [and] each qubit connected to 15 others -- so, that will allow us to solve significantly larger problems.

In fact, for a doubling of the processor size, you get about a tripling of the problem size. But we've done one other thing: We've developed brand new hybrid algorithms [and] these are not types of hybrid algorithms that people typically think about. It's not like you try to take the problem and divide it into chunks and solve [them]. This is a hybrid approach where we use a classical technique to find the core of the problem, and then we send that off to the quantum processor. With that, we can get to the point where we can solve large problems even with our 5,000-qubit system. We think once we deliver the 5,000-qubit system Advantage [and] the new hybrid solver, we'll see more and more companies being able to solve real production problems.

Do you think companies are in a race toward quantum computing?

Baratz: No, they're not because in some cases they're not aware, and in some cases they don't understand what's possible. The problem is there are very large companies that make a lot of noise in the quantum space and their approach to quantum computing is one where it will take many, many years before companies can actually do something useful with the system. And because they're the loudest out there, many companies think that's all there is. We've been doing a better job of getting the D-Wave message out, but we still have a ways to go. What excites you most about quantum comput- ing in business settings?

Baratz: First, just the ability to solve problems that can't otherwise be solved to me is exciting. When I was at MIT, my doctorate was in theory of computation. I was kind of always of the mindset that there are just some problems that you're not going to be able to solve with one computer. I wouldn't say quantum computing removes, but [it] reduces that limitation -- that restriction -- and it makes it possible to solve problems that couldn't otherwise be solved.

But more than that, the collection of technologies that we have to use to build our system, even that is exciting. As I mentioned during the panel, we develop new superconducting circuit fabrication recipes. And we're kind of always advancing the state of the art there. We're advancing the state of the art and refrigeration and how to remove contaminants from refrigerators because it can take six weeks to calibrate a quantum computer. Once you cool it down, would you cool the chip down? Well, if you get contaminants in the refrigerator and you have to warm up the system and remove those contaminants, you're going to lose two months of compute time. We have systems that run for two and a half, three years and nobody else really has the technology to do that.

It's been said that one of the main concerns with quantum computers is increased costs. Can you talk a little more about that?

Baratz: Well, there was that power question [from the panel] about when is it going to stop all the power? So, the truth of the matter is our system runs on about 20 kilowatts. The reason is, the only thing we really need power for is the refrigerator -- and we can put many chips in one refrigerator. So, as the size of the chip grows, as the number of chips grow, the power doesn't grow. Power is the refrigerator.

Second, the systems are pricey if you want to buy one [but through] cloud access, anybody can get it. I mean, we even give free time, but we do sell it and currently we sell it at $\$ 2,000$ an hour, but you can run many, many problems within that amount of time 